\title{
Early results from AIRS/AMSU/HSB
}

\author{
Joel Susskind ${ }^{\mathrm{a}}$, Christopher Barnet ${ }^{\mathrm{b}}$, John Blaisdell $^{\mathrm{c}}$, Lena Iredell ${ }^{\mathrm{c}}$, Fricky Keita ${ }^{\mathrm{c}}$, Lou Kouvaris ${ }^{\mathrm{c}}$ \\ ${ }^{a}$ NASA Goddard Space Flight Center, Greenbelt, MD, USA 20771 \\ bJoint Center for Earth Systems Technology, NASA GSFC, Greenbelt, MD, USA 20771 \\ 'Science Applications International Corporation, NASA GSFC, Greenbelt, MD, USA 20771
}

\begin{abstract}
AIRS was launched on EOS Aqua on May 5, 2002, together with AMSU A and HSB, to form a next generation polar orbiting infrared and microwave atmospheric sounding system. The primary products of AIRS/AMSU/HSB are twice daily global fields of atmospheric temperature-humidity profiles, ozone profiles, sea/land surface skin temperature, and cloud related parameters including OLR. The sounding goals of AIRS are to produce $1 \mathrm{~km}$ tropospheric layer mean temperatures with an rms error of $1 \mathrm{~K}$, and layer precipitable water with an rms error of $20 \%$, in cases with up to $80 \%$ effective cloud cover. Pre-launch simulation studies indicated that these results should be achievable. Minor modifications have been made to the pre-launch retrieval algorithm as described in this paper. Sample fields of parameters retrieved from AIRS/AMSU/HSB data are presented and validated as a function of retrieved fractional cloud cover. As in simulation, the degradation of retrieval accuracy with increasing cloud cover is small. Select fields are also compared to those contained in the ECMWF analysis, done without the benefit of AIRS data, to demonstrate information that AIRS can add to that already contained in the ECMWF analysis.
\end{abstract}

Keywords: Infra red, microwave, next generation, sounding

\section{INTRODUCTION}

AIRS/AMSU/HSB is a state of the art advanced infra-red microwave sounding system that was launched on the EOS Aqua platform in a 1:30 AM/PM sun synchronous orbit on May 5, 2002. An overview of the AIRS instrument and the objectives for AIRS/AMSU/HSB is a given in Aumann et al ${ }^{1}$. The sounding goals of AIRS are to produce $1 \mathrm{~km}$ tropospheric layer mean temperatures with an rms error of $1 \mathrm{~K}$, and layer precipitable water with an rms error of $20 \%$, in cases with up to $80 \%$ effective cloud cover. Aside from being part of a climate mission, one of the objectives of AIRS is to provide sounding information of sufficient accuracy such that when assimilated into a general circulation model, significant improvement in forecast skill would arise. The pre-launch algorithm to produce level 2 products (geophysical parameters) using AIRS/AMSU/HSB data, and expected results based on simulation studies, are given in Susskind et al. ${ }^{2}$ The results of this simulation indicate that the sounding goals of AIRS/AMSU/HSB should be achievable. In this simulation, perfect knowledge of the instrumental spectral response functions and the inherent physics of the radiative transfer equations was assumed. Therefore, if the true state of the atmosphere and underlying surface were known perfectly, one could compute the radiances AIRS, AMSU, and HSB would see exactly up to instrumental noise. Susskind et al. ${ }^{2}$ alluded to the fact that this is not the case in reality, and additional terms would have to be included in the retrieval algorithm to account for systematic differences (biases) between observed brightness temperatures and those computed knowing the "true" surface and atmospheric state, as well as residual computational errors after that systematic bias is accounted for (computational noise). In this paper, we will show results based on the algorithm we were using to analyze AIRS/AMSU/HSB data on June 30, 2003, which we will refer to as Version 3.1. This algorithm is very similar to the pre-launch version, with the major differences attributable to the factors described above. JPL delivered an earlier version of the algorithm to the Goddard DAAC, Version 3.0, for the earliest near real time processing of AIRS level 2 products starting in July 2003. In this paper, we will briefly describe the differences between the pre-launch version, Version 3.0, and Version 3.1, and show sample results using Version 3.1 on data for September 6, 2002, and January 25, 2003. Research to further improve the results of analysis of AIRS/AMSU/HSB data is continuing. 


\section{DIFFERENCES BETWEEN THE PRE-LAUNCH ALGORITHM, VERSION 3.0, AND VERSION 3.1}

The differences between all three versions of the retrieval algorithm are relatively small. The post-launch channel frequencies were somewhat different from those pre-launch, as expected, as were the channel spectral response functions. Consequently, new Radiative Transfer Algorithm (RTA) coefficients were generated ${ }^{3}$ to be consistent with the post-launch instrumental conditions. Minor modifications were therefore made to the set of channels used in the retrieval algorithm. The most significant of these resulted from the finding that more channels in the $4.3 \mu \mathrm{m}$ region were affected by non-local thermodynamic equilibrium (non-LTE) than previously thought (see Figure 1b). Radiances in these channels are perturbed during the day, and these channels are currently not used in the retrieval algorithm day or night. It was also found that observed channel brightness temperatures for AIRS, as well as AMSU and HSB, were biased from those computed using the RTA with the best estimate of the truth. These biases, referred to as "tuning coefficients", are subtracted from all terms in the retrieval algorithm involving observed minus computed brightness temperatures. New regression coefficients were also generated ${ }^{4}$ based on clear column radiances for an ensemble of accepted retrievals, using the 3 hour ECMWF forecast at "truth".

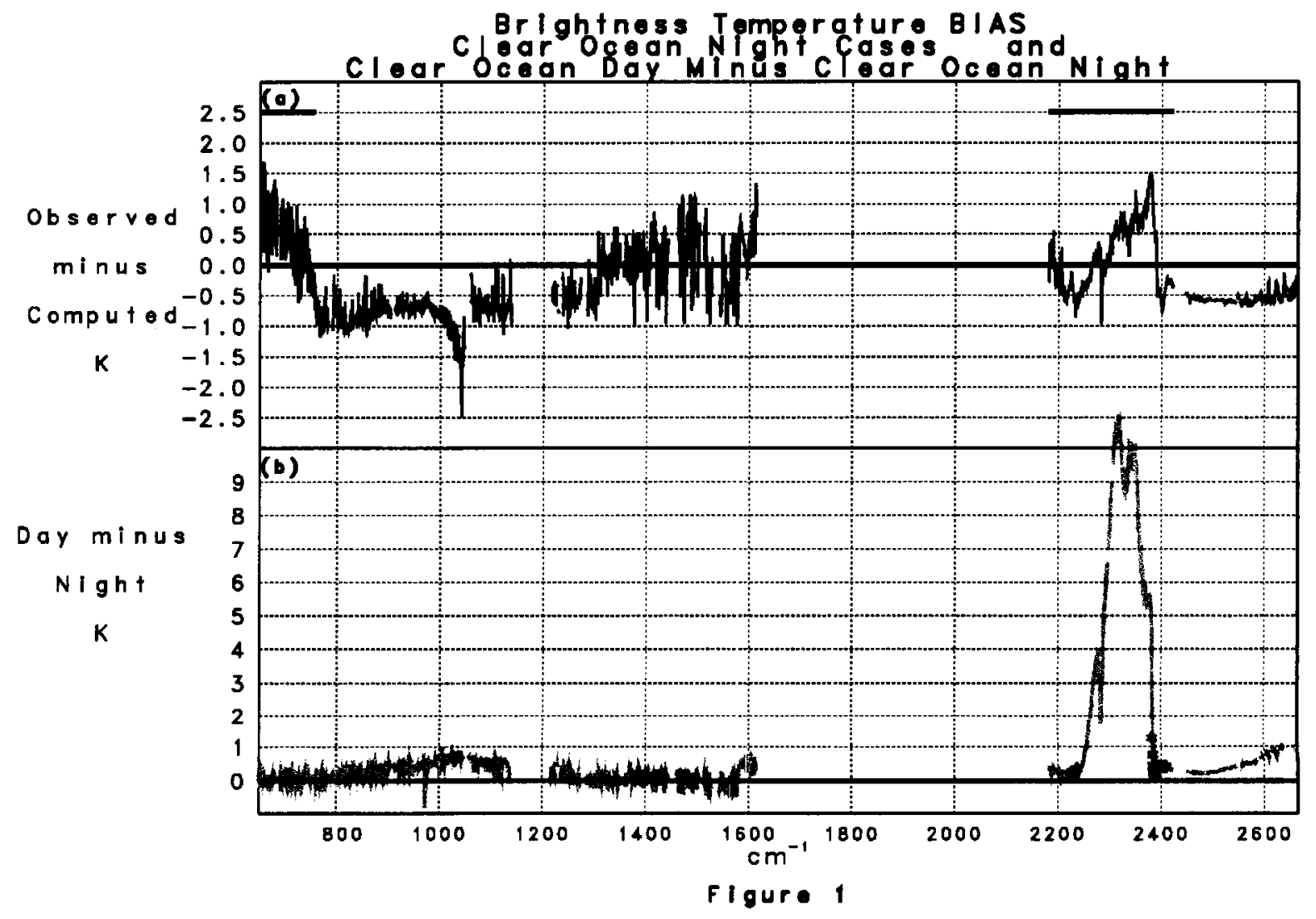

\subsection{Generation of tuning coefficients}

In order to generate tuning coefficients, we select cases thought to be unaffected by clouds so as not to have to account for cloud effects on the observed radiances. As with the generation of regression coefficients, we use the 3 hour ECMWF forecast, collocated to the satellite observations, as truth, and limit the observations to nighttime ocean, $40^{\circ} \mathrm{N}-$ $40^{\circ} \mathrm{S}$. Ocean cases were selected because we have the best estimate of both sea-surface temperature and surface emissivity over oceans compared to land. For the computation of IR biases, we selected cases for which the retrieval was accepted and called essentially clear ${ }^{2}$. Figure 1a shows the nighttime biases of observed minus computed brightness temperatures for the AIRS channels. These biases had very little zenith angle dependence. Computed biases in channels affected significantly by radiation emitted from the surface are less meaningful because of uncertainty in the 
surface skin temperature and emissivity. Likewise, biases for channels significantly effected by ozone absorption are suspect because of limited accuracy of ozone profiles in the ECMWF forecast, and to a lesser extent, this holds for channels significantly affected by water vapor absorption as well. In general, observed brightness temperatures are somewhat warmer (generally $0.5 \mathrm{~K}-1 \mathrm{~K}$ ) than those computed using the RTA in the $\mathrm{CO}_{2}$ absorption region $650 \mathrm{~cm}^{-1}-$ $710 \mathrm{~cm}^{-1}$. Figure $1 \mathrm{~b}$ shows that daytime biases are very similar to nighttime biases, except for the region between 2240 $\mathrm{cm}^{-1}$ and $2384 \mathrm{~cm}^{-1}$. Daytime radiances in this region are affected to varying degrees by non-LTE. Figure $1 \mathrm{~b}$ indicates by stars the channels currently used for temperature sounding in the spectral region $2200 \mathrm{~cm}^{-1}-2420 \mathrm{~cm}^{-1}$, which is a smaller set than in the pre-launch version. Tuning coefficients are used only for channels in the range $650 \mathrm{~cm}^{-1}-756$ $\mathrm{cm}^{-1}$ ( $\mathrm{CO}_{2}$ absorption), and $2180 \mathrm{~cm}^{-1}-2422 \mathrm{~cm}^{-1}\left(\mathrm{~N}_{2} \mathrm{O}\right.$, and $\mathrm{CO}_{2}$ absorption). Those spectral regions in which the tuning coefficients are used are indicated by the horizontal bars in Figure 1. To first order, these tuning coefficients remove biases between observed and computed brightness temperatures, but are not accurate for every situation. As a crude estimate of residual error, called "computational error", we took half the bias for each AIRS channel. These computational errors are added to the diagonal term of the channel noise covariance matrix in each retrieval step for all channels, whether they are tuned or not.

For the computation of microwave bias, a similar set of cases was selected, but only scenes with precipitating clouds were excluded from the ensemble used to compute the bias. With regard to AMSU A, there is a known calibration problem in that the current calibration algorithm does not take into account effects of side-lobes (which can see cold space) on the observed radiances. This gives rise to large (many degrees), highly zenith angle dependent, channel biases. Therefore, for microwave channels, we compute a separate bias for each channel and beam position. This bias is not used for the HSB channels because the side-lobe correction is smaller in the HSB than in AMSU A, as well as the concerns about the accuracy of the ECMWF water vapor field, as discussed above.

\subsection{Rejection criteria thresholds}

Rejection criteria thresholds were loosened in analysis of real data compared to what was felt optimal based on simulation $^{2}$ so as to increase the percent of accepted retrievals. Many of the changes are necessitated by the fact that the microwave first product is poorer, primarily as a result of residual errors in the microwave channel bias corrections. The threshold $\Delta \mathrm{F}$, representing the ability to find a set of cloud cleared radiances which are consistent with what is expected from the microwave only retrieval, was increased (larger differences allowed) from 1.75 to 4.0. We also now allow residuals in either the temperature profile retrieval or the surface skin temperature retrieval to be as much as 4 times that predicted by the channel noise covariance matrix, rather than being not greater than that value. We currently accept more difficult cloud cases as well, allowing the noise amplification factor to be as great as 5.0, rather than 2.0. Version 3.0 does not check the effective noise amplification factor, $A_{\text {eff }}$, at all (maximum value 8 in the pre-launch version). We have found $A_{\text {eff }}$ to be a strong indicator of the accuracy of the temperature profile in the lowest $3 \mathrm{~km}$, as well as of sea surface temperature over ocean. In Version 3.1, the retrieval is rejected if $\mathrm{A}_{\text {eff }}>10$ over ocean, and flagged as "questionable" in the lowest $3 \mathrm{~km}$ over land if $\mathrm{A}_{\mathrm{eff}}>10$. In addition, Version 3.1 significantly increased the yield over cold surfaces by doubling all rejection thresholds for cases with skin temperature less than $265 \mathrm{~K}$. Both Versions 3.0 and 3.1 added an additional gross error check, rejecting the retrieval over non-frozen ocean if the retrieved sea-surface temperature differs from its expected value, provided in the ECMWF forecast, by more than $3 \mathrm{~K}$.

\section{RESULTS USING VERSION 3.1}

Figure 2 shows the number of cases for each retrieved effective fractional cloud for the whole day September 6, 2002. The effective fractional cloud cover is given by the product of the fraction of the field of view covered by clouds and the cloud emissivity at $11 \mu \mathrm{m}$. The average global effective cloudiness was determined to be $38.13 \%$. Also shown is the percent of accepted retrievals as a function of retrieved effective cloud cover. Roughly $85 \%$ of the cases with retrieved effective cloud cover $20 \%$ were accepted, falling to $70 \%$ at $60 \%$ effective cloud cover, and to $50 \%$ at $80 \%$ effective cloud cover. All cases with retrieved effective cloud cover greater than $80 \%$ are rejected. ${ }^{2}$ The average effective fractional cloudiness for all accepted cases was $29.97 \%$. These results are very similar to what was shown in the simulation study. ${ }^{2}$ 


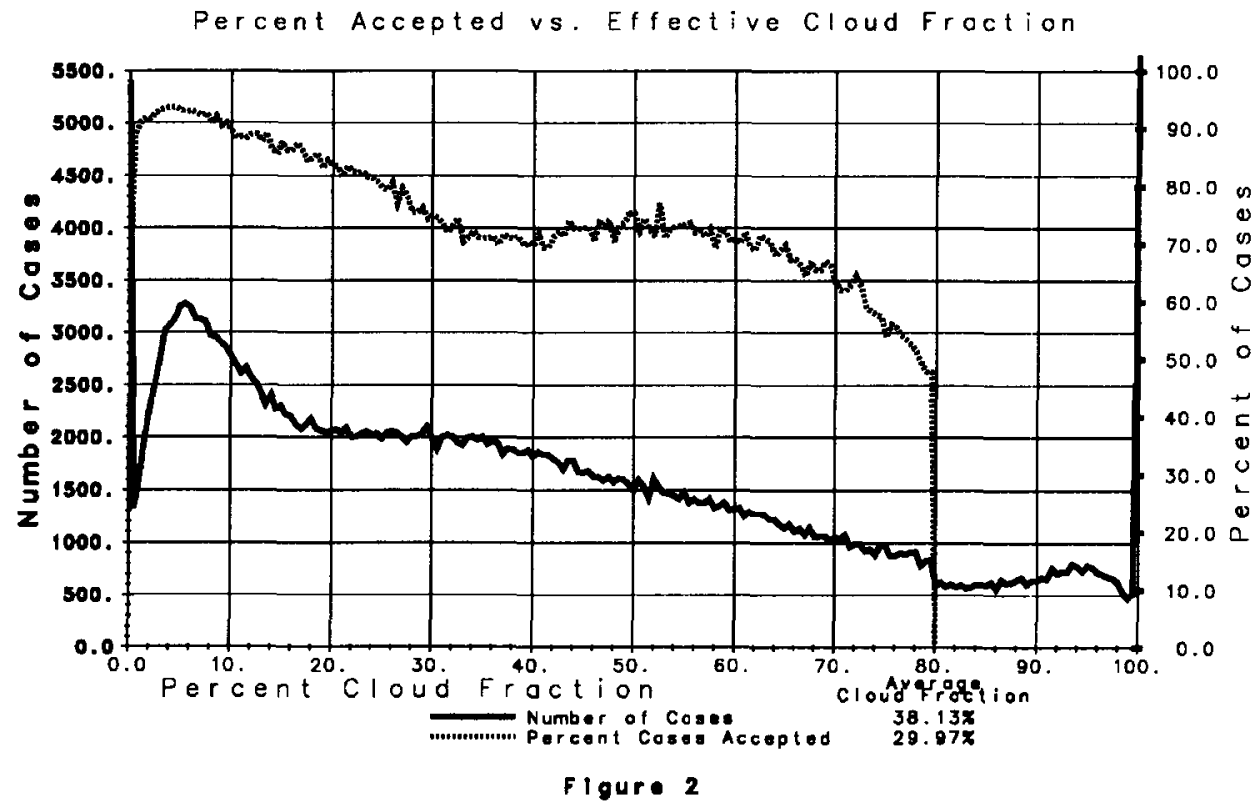

Figure 3 shows the RMS difference between retrieved $1 \mathrm{~km}$ layer mean temperatures and the collocated ECMWF forecast for all accepted cases as a function of retrieved effective cloud fraction. Results are shown for each of the lowest $8 \mathrm{~km}$ of the atmosphere. Agreement degrades with increasing cloud cover, but only very slowly except in the lowest $2 \mathrm{~km}$ of the atmosphere. RMS temperature differences from ECMWF at all levels are somewhat larger than the 1 $\mathrm{K}$ goal for retrieval accuracy. Part of this difference can be attributed to the fact that the ECMWF forecast is not perfect. It is also possible that the accuracy of the ECMWF forecast may be somewhat poorer with increasing cloud cover. RMS differences from ECMWF grow significantly with effective cloud fraction greater than $40 \%$ in the two lowest $1 \mathrm{~km}$ layers. Based on this finding, Version 3.1 currently flags the retrievals in the lowest $3 \mathrm{~km}$ as "questionable" for retrieved effective cloud cover greater than $40 \%$.

AIRS RMS Temperoture Differences from ECMWF vs. Cloudiness

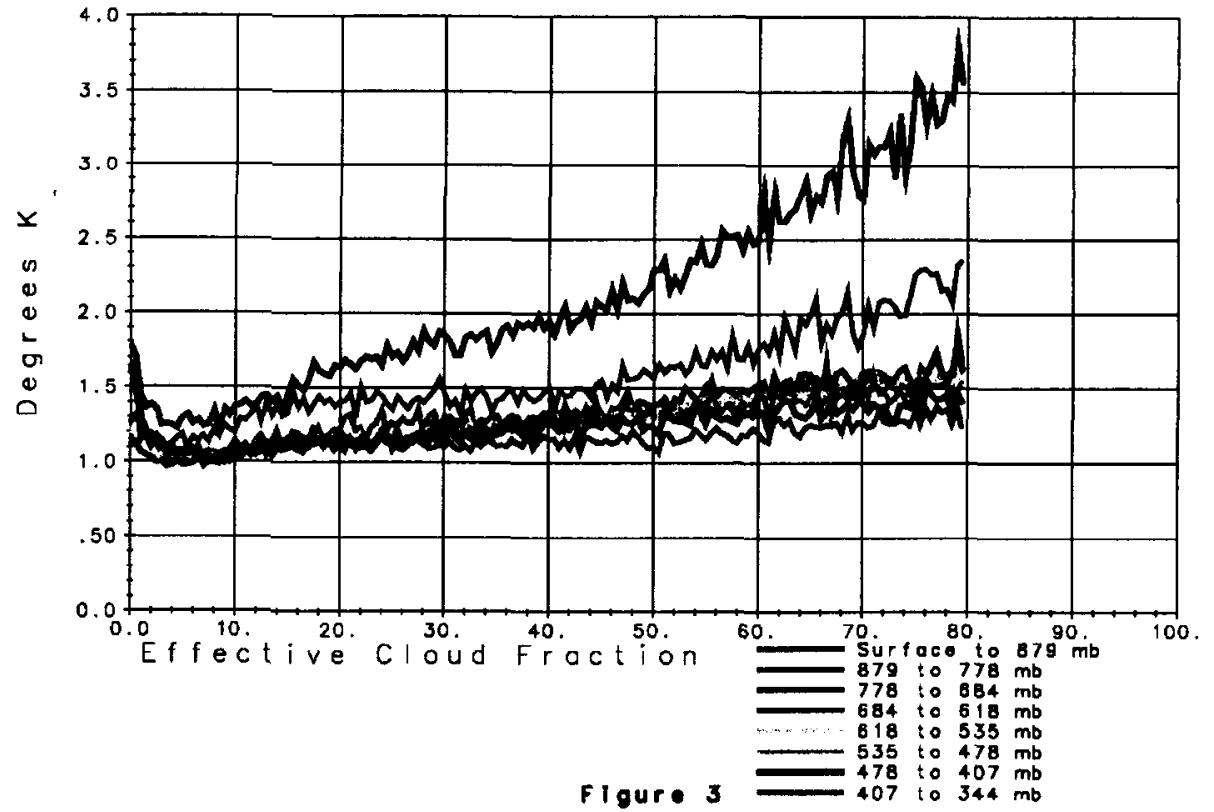


Figure 4a shows RMS layer mean temperature differences between accepted retrievals, the ECMWF forecast, and collocated radiosonde reports $( \pm 1$ hour, $\pm 100 \mathrm{~km}$ ) for September 6, 2002. The layers, which are roughly $1 \mathrm{~km}$ thick up to $100 \mathrm{mb}$, and $3 \mathrm{~km}$ thick above that, are indicated in the figure. The "questionable" retrieved temperatures in the lowest $3 \mathrm{~km}\left(\mathrm{~A}_{\text {eff }}>10\right.$ or effective cloud fraction $\left.>40 \%\right)$ are excluded in generating the statistics shown. The number of cases included in each of (a subset of) the layers is indicated at the right of the figure. It is interesting to note that the RMS differences between the retrievals and ECMWF are smaller in the vicinity of radiosondes than they were globally (see Figure 3). In particular, in the lowest $1 \mathrm{~km}$, the RMS difference is $1.1 \mathrm{~K}$, rather than a number closer to $2 \mathrm{~K}$ that would be inferred from Figure 3. This is because the ECMWF forecast is more accurate in the vicinity of radiosondes than it is globally. The 3 hour ECMWF forecast agrees with radiosondes to $1 \mathrm{~K}$ between roughly $750 \mathrm{mb}$ and $20 \mathrm{mb}$. Spatial and temporal sampling differences between ECMWF, retrievals, and radiosondes contribute to some extent to the increased differences between both ECMWF and retrievals as compared to radiosondes beneath $750 \mathrm{mb}$, as spatial and temporal variability of the atmosphere is greatest near the surface. Retrieval accuracy near radiosondes is somewhat poorer than that of the forecast at all levels, especially in the vicinity of $200 \mathrm{mb}$. This is most likely due to limitations in the current tuning coefficients and noise covariance matrix. We expect further improvement in this area.

Figure $4 \mathrm{~b}$ shows analogous results for percent differences in $1 \mathrm{~km}$ layer mean precipitable water, for which the sounding goals for AIRS is $20 \%$. With regard to water vapor, it is clear that AIRS retrievals are significantly more accurate than the ECMWF forecast above $700 \mathrm{mb}$. AIRS differences from radiosondes are greater than the $20 \%$ goal. Spatial and temporal sampling differences between AIRS and radiosondes may contribute significantly to the apparent water vapor "errors" as water vapor changes rapidly in space and time.

LAYER MEAN RMS TEMPERATURE ( $\mathrm{C}$ ) Sept amb or 6,2002

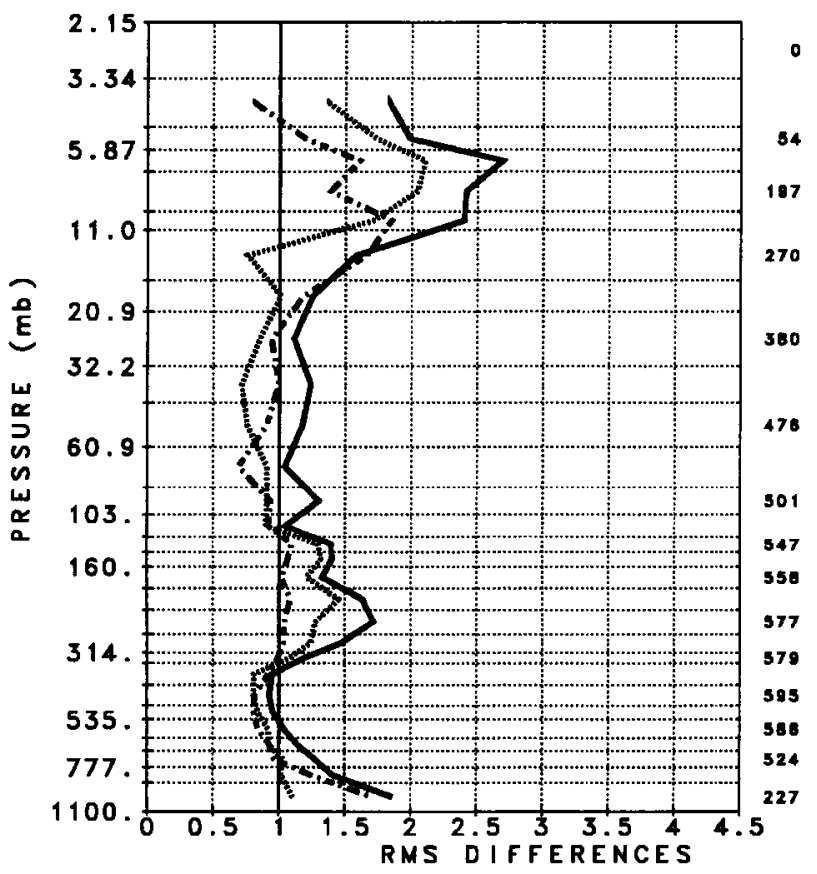

$1 \mathrm{Km}$ LAYER PRECIPITABLE WATER September 6, 2002
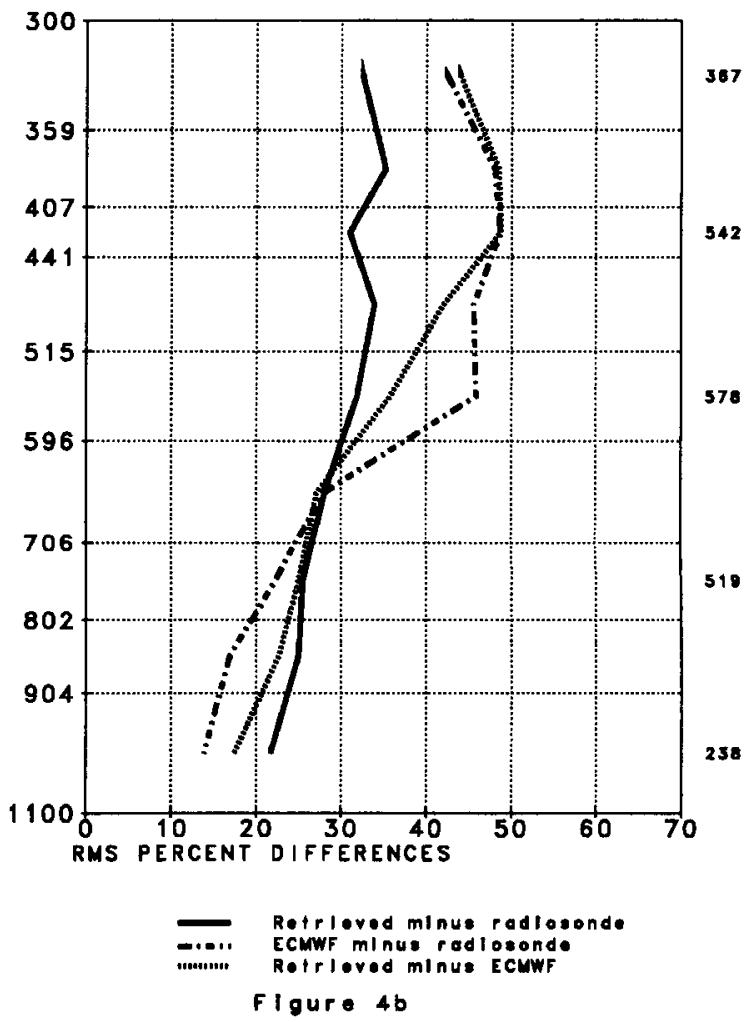
Figures 5a-d show sample retrieved fields gridded into $1^{\circ} \times 1^{\circ}$ latitude-longitude bias for ascending (daytime) orbits on September 6. Missing data (no retrievals in a bin) is shown as gray. Retrieved cloud parameters are shown in Figure 5a. The height of the clouds is indicated by the color and the effective cloud fraction by the intensity, with faint colors indicating effective cloud fraction less than $20 \%$ and the brightest colors greater than $80 \%$. Low clouds are shown in yellow and orange, high clouds in red and purple, and mid-level clouds in green to violet. Cloud parameters are always retrieved, so gray means no satellite data was observed. Figure $5 \mathrm{~b}$ shows the retrieved $700 \mathrm{mb}$ temperature field, representative of the lower troposphere, for the same time period. Gray indicates either no data observed or all retrievals were rejected in the grid box. In addition, elevated terrain in which the surface pressure is less than $700 \mathrm{mb}$ are indicated as having no data. Elevated terrain is not a factor in Figures $5 \mathrm{c}$ and $5 \mathrm{~d}$, which are at lower pressures. In most cases, additional missing areas coincide with cloud cover greater than $80 \%$ (the brightest colors in Figure $5 \mathrm{a}$ ). The retrieved $700 \mathrm{mb}$ temperature field is spatially coherent, even in the presence of relatively large amounts of cloud cover. The areas of missing data usually occur at the leading edge of cold fronts, where cold air masses join warmer ones. Figure 5c shows the total precipitable water vapor above $300 \mathrm{mb}$, corresponding to upper tropospheric water vapor. This field is not well measured by any source other than AIRS and is a very important factor in the Greenhouse effect and the response of atmospheric temperatures to increases in trace gas (such as $\mathrm{CO}_{2}$ ) concentrations. Upper tropospheric water vapor is largest in the vicinity of high clouds. We do not retrieve upper tropospheric water vapor under extensive cloud cover, but do retrieve it in surrounding areas. Figure 5c shows that upper tropospheric water vapor is spatially much more variable than temperature. In addition, high values of upper tropospheric water vapor occur ahead (on the warm air side) of the clouds associated with cold fronts, and low values appear behind the clouds. Figure $5 \mathrm{~d}$ shows retrieved temperatures at $100 \mathrm{mb}$, representative of the lower stratosphere. A comparison of Figures $5 \mathrm{~b}$ and $5 \mathrm{~d}$ show that thermal features in the lower troposphere and lower stratosphere tend to be out of phase with each other. Note in particular the features north of $30^{\circ} \mathrm{N}$, in which cold air masses in the lower troposphere correspond to warmer air in the lower troposphere.
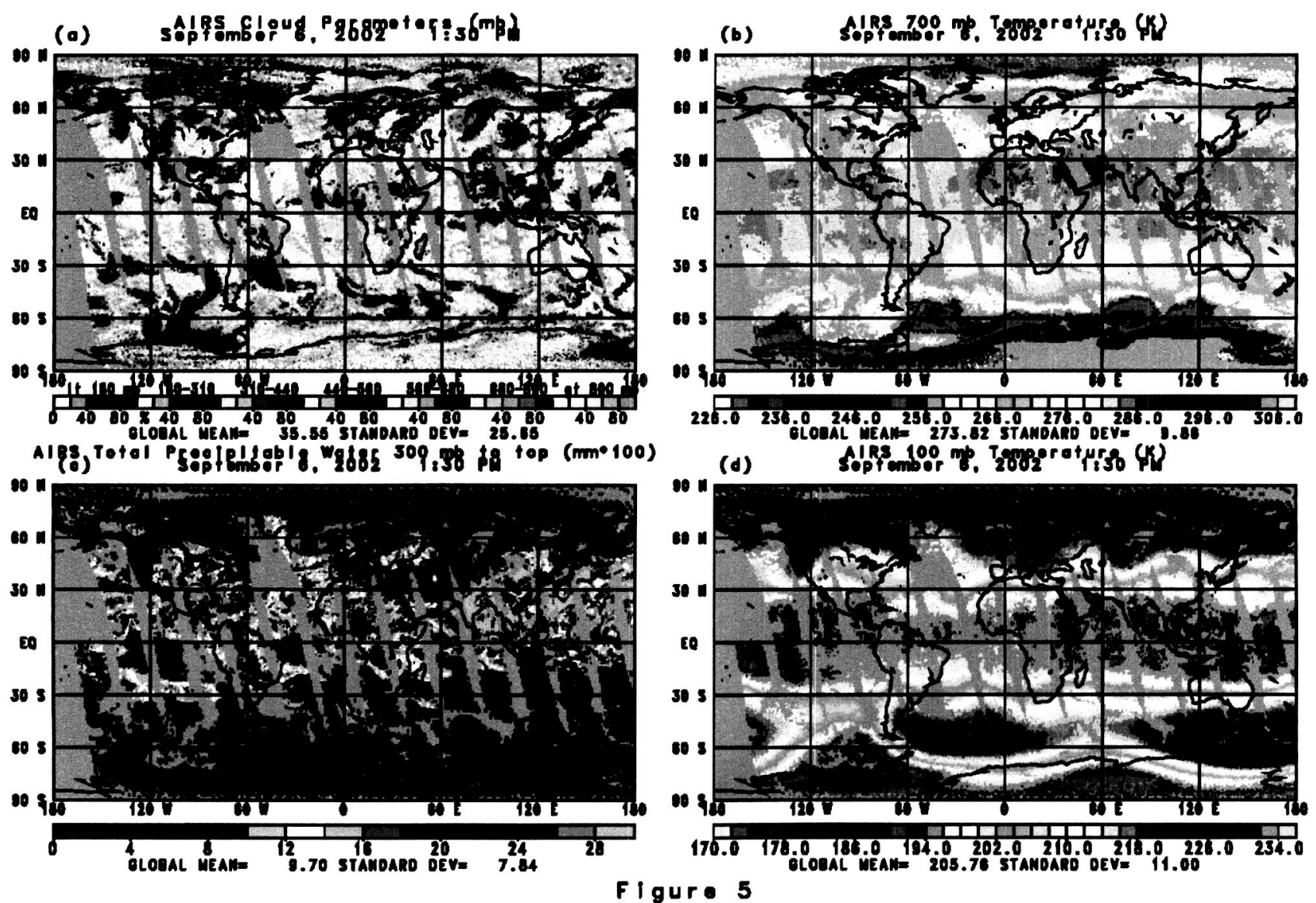
Figure 6a shows the retrieved $700 \mathrm{mb}$ temperature field for ascending orbits on January 25,2003 . Figure $6 \mathrm{~b}$ shows the collocated ECMWF 3 hour forecast $700 \mathrm{mb}$ temperature field. These fields appear very similar to each other. Their difference is shown in Figure $6 \mathrm{c}$, in which white shows agreement to $\pm 0.5 \mathrm{~K}$, each shade of red shows AIRS warmer than ECMWF in intervals of $1 \mathrm{~K}(0.5-1.5,1.5-2.5$, etc.), and each shade of blue shows AIRS colder then ECMWF in intervals of $1 \mathrm{~K}$. The area weighted global mean difference of the two fields is $0.08 \mathrm{~K}$, and the area weighted standard deviation is $1.13 \mathrm{~K}$. Most areas are white or the first shade of red or blue. The largest differences between the two fields occur in the vicinity of $35^{\circ} \mathrm{S}-55^{\circ} \mathrm{S}, 100^{\circ} \mathrm{E}-140^{\circ} \mathrm{E}$, and show up as a dipole, with AIRS warmer to the west of $120^{\circ} \mathrm{E}$ and colder to the east. Figures $6 \mathrm{a}$ and $6 \mathrm{~b}$ show this to be an area of a cold air mass, extending from the polar region to the mid-latitudes. This cold air mass is coherent in both the retrieved and forecasted fields, but is centered further east in the retrieved field compared to the forecast field, corresponding to a phase error in the 3 hour ECMFW forecast. This is precisely the type of information that satellite data can provide (if accurate enough) to help improve forecast skill. Figure $6 \mathrm{~d}$ shows the difference between the retrieved and forecasted $100 \mathrm{mb}$ temperature fields. At the $100 \mathrm{mb}$ level, a corresponding warm front (not shown) exists in both the retrieved and forecasted fields in the area discussed above, with an analogous phase error to that found at $700 \mathrm{mb}$. Consequently, the retrieved $100 \mathrm{mb}$ field is cooler than ECMWF to the west and warmer to the east in the region discussed above. This out of phase relationship of patterns of differences from ECMWF at $700 \mathrm{mb}$ and $100 \mathrm{mb}$ is indicative of phase errors in the ECMWF forecast, as there is no reason for retrieval errors to be out of phase with each other at $700 \mathrm{mb}$ and $100 \mathrm{mb}$. This out of phase relationship in spatial patterns of differences between retrieved and forecast temperatures at $700 \mathrm{mb}$ and $100 \mathrm{mb}$ is found in numerous places in Figures $6 \mathrm{c}$ and $6 \mathrm{~d}$ and indicate many areas where the satellite data should improve the ECMWF forecast. We plan an experiment in the near future to assimilate AIRS retrievals for all of January 2003 into the GSFC Data Assimilation Office General Circulation model to assess improvement in forecast skill.
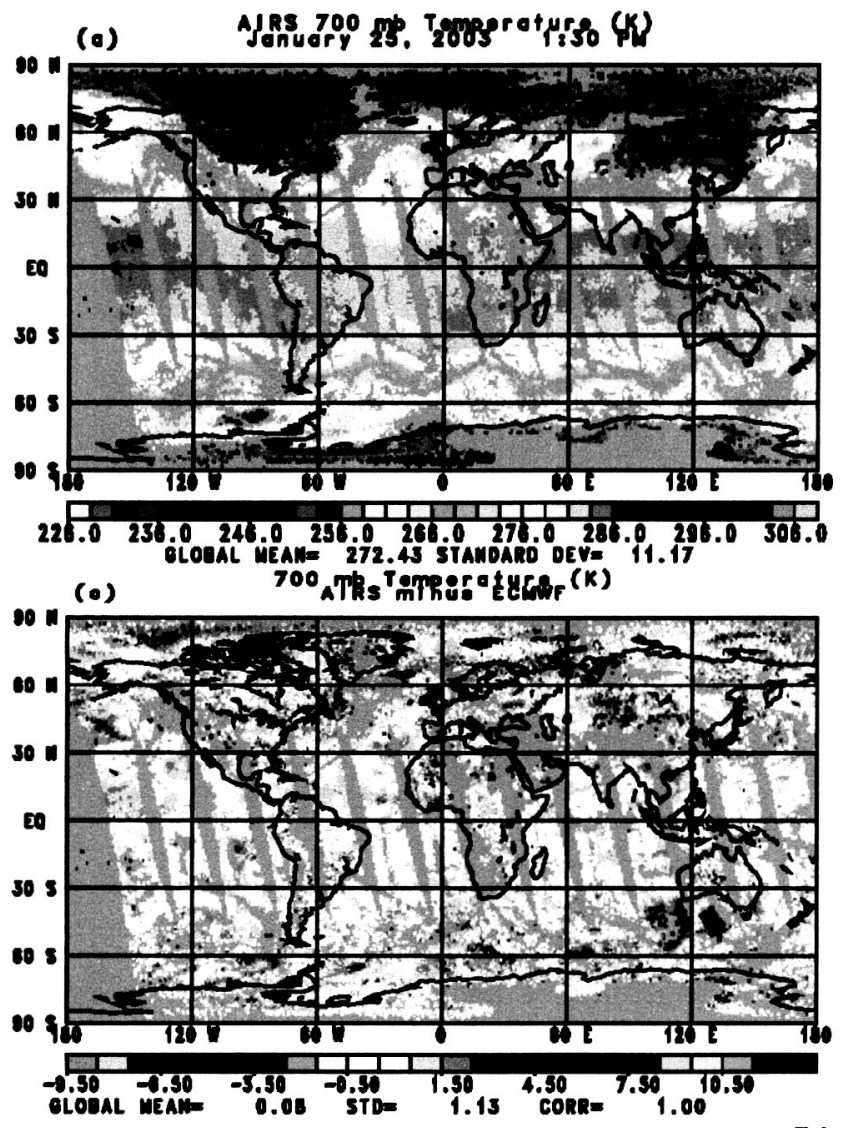

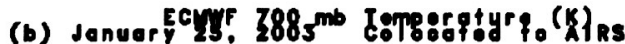
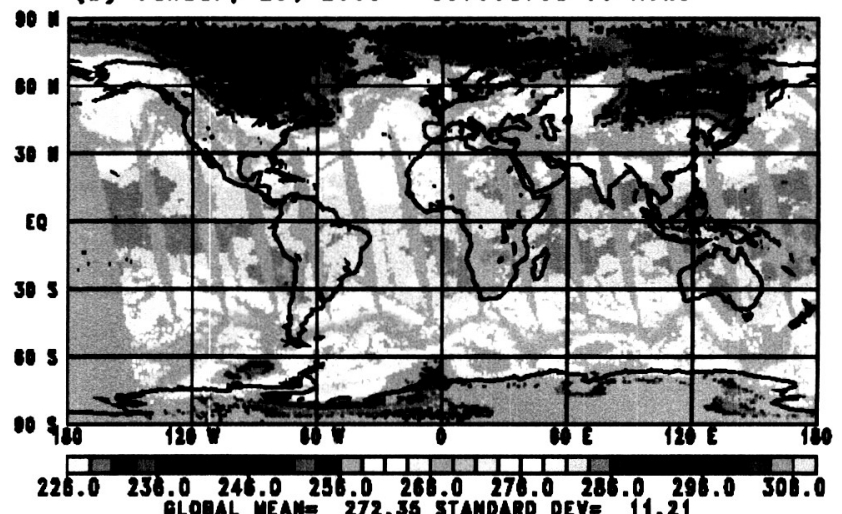
(d)

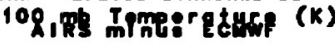

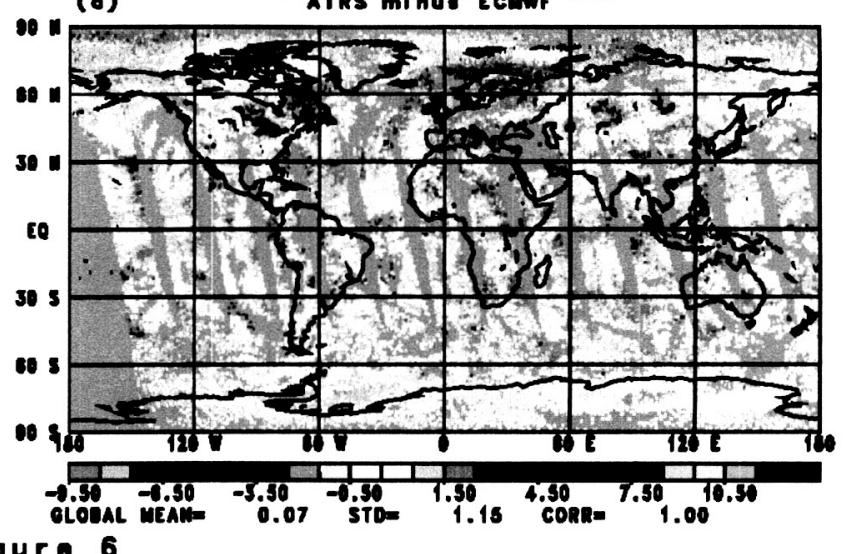




\section{REFERENCES}

1. Hartman H. Aumann, Moustafa T. Chahine, Catherine Gautier, Mitchell D. Goldberg, Eugenia Kalnay, Larry M. McMillin, Hank Revercomb, Philip W. Rosenkranz, William L. Smith, David H. Staelin, L. Larrabee Strow, and Joel Susskind, "AIRS/AMSU/HSB on the Aqua Mission: Design, Science Objectives, Data Products, and Processing Systems". IEEE Trans. On Geoscience and Remote Sensing, 41, 253-264, 2003.

2. Joel Susskind, Christopher B. Barnet, and John M. Blaisdell, "Retrieval of Atmospheric and Surface Parameters from AIRS/AMSU/HSB Data in the Presence of Clouds". IEEE Trans. On Geoscience and Remote Sensing, 41, 390-409, 2003.

3. Mitchell D. Goldberg, Yanni Qu, Larry M. McMillin, Walter Wolf, and Murty Divakarla, "AIRS Near-Real-Time Products and Algorithms in Support of Operational Numerical Weather Prediction". IEEE Trans. on Geoscience and Remote Sensing, 41, 379-389, 2003.

4. L. Larrabee Strow, Scott E. Hannon, Sergio DeSouza-Machado, Howard E. Motteler, and David Tobin, "An Overview of the AIRS Radiative Transfesr Model". IEEE Trans. on Geoscience and Remote Sensing, 41, 303-313, 2003. 
Early Results from AIRS/AMSU/HSB

\author{
Dr. Joel Susskind \\ Laboratory for Atmospheres \\ NASA Goddard Space Flight Center
}

\begin{abstract}
Popular Summary
This is an extended abstract for a paper to be presented at the International Symposium on Optical Science and Technology entitled "Early results from AIRS/AMSU/HSB."

AIRS/AMSU/HSB is an advanced IR/microwave atmospheric sounding system launched on the Eos Aqua satellite on May 5, 2002. The goals of AIRS/AMSU/HSB are to produce atmospheric soundings of temperature profile with RMS errors of tropospheric $1 \mathrm{~km}$ layer mean temperatures of $1 \mathrm{~K}$, and $1 \mathrm{~km}$ layer integrated precipitable water vapor of $20 \%$, in cases of up to $80 \%$ cloudiness. Soundings with this accuracy are expected to be able to significantly improve forecast skill. The status of AIRS soundings, as of June 30, 2003, is presented. Results show that accurate AIRS soundings can be obtained in up to $80 \%$ cloud cover, and that AIRS adds significant information to about the state of the atmosphere to a 3 hour ECMWF.
\end{abstract}

